Check for updates

Cite this: Chem. Sci., 2019, 10, 6330

๑ All publication charges for this article have been paid for by the Royal Society of Chemistry

\section{Stable and tunable plasmon resonance of molybdenum oxide nanosheets from the ultraviolet to the near-infrared region for ultrasensitive surface-enhanced Raman analysis $\dagger$}

\author{
Jinhu Wang, ${ }^{a}$ Yinhua Yang, ${ }^{\mathrm{b}}$ Hua Li, ${ }^{\mathrm{b}}$ Jun Gao, ${ }^{\mathrm{c}}$ Ping He, ${ }^{\mathrm{c}}$ Liang Bian, ${ }^{\mathrm{d}}$ Faqin Dong ${ }^{\mathrm{d}}$ \\ and $\mathrm{Yi} \mathrm{He}_{\mathrm{BD}}$ *a
}

Preparation of color-tunable and stable plasmonic $\mathrm{MoO}_{3}$ nanomaterials remains challenging, due to the lack of an effective preparation strategy and surface protection in heavily doped $\mathrm{MoO}_{3}$. Herein, we report a facile and reliable method for synthesis of oxygen-deficient $\mathrm{MoO}_{3}\left(\mathrm{MoO}_{3-x}\right)$ nanosheets using dopamine as the reducing agent and precursor for the formation of a polydopamine (PDA) surface coating. The PDA-coated $\mathrm{MoO}_{3-x}$ nanosheets show stable and tunable localized surface plasmon resonance (LSPR) from the ultraviolet to the near-infrared region (361-809 $\mathrm{nm}$ ) via altering the $\mathrm{pH}$ value of the medium, accompanying the generation of multicolor nanosheet dispersions, such as deep blue, faint bluish, orange, yellow and black. Importantly, the resulting PDA-coated $\mathrm{MoO}_{3-x}$ nanosheets are quite stable even in the presence of oxidants, and they can be used as an ultrasensitive surfaceenhanced Raman scattering (SERS) substrate. The limit of detection for rhodamine 6G (R6G) dye is down to $0.3 \mathrm{fM}$ concentration, and the corresponding Raman enhancement factor reaches $1 \times 10^{10}$. The coupling of charge transfer between R6G and PDA-coated $\mathrm{MoO}_{3-x}$ nanosheets and molecular resonances may be responsible for the strong SERS effect.
Received 7th May 2019

Accepted 17th May 2019

DOI: $10.1039 / c 9 s c 02202 c$

rsc.li/chemical-science

\section{Introduction}

Plasmonic nanomaterials with distinct localized surface plasmon resonance (LSPR) features have been widely studied in the past decade owing to their promising applications in chemical sensing, catalysis, molecular imaging, optical waveguides, color displays, $\mathrm{CO}_{2}$ reduction, and $\mathrm{N}_{2}$ fixation. ${ }^{1-8}$ Conventional plasmonic nanomaterials are based on noble metals because they have stable properties and high carrier density. ${ }^{9,10}$ Additionally, the LSPR of noble metals is tunable from the visible to the nearinfrared range through shape, size, and composition control. ${ }^{11}$ On the other hand, the LSPR property is also discovered in doped metal oxide semiconductor nanocrystals such as tin

${ }^{a}$ State Key Laboratory of Environment-friendly Energy Materials, Sichuan Co-Innovation Center for New Energetic Materials, Southwest University of Science and Technology, Mianyang 621010, P. R. China. E-mail: yhe2014@126.com

${ }^{b}$ Materials Characterization \& Preparation Center, Southern University of Science and Technology, Shenzhen 518055, China

${ }^{c}$ School of Materials Science and Engineering, Southwest University of Science and Technology, Mianyang, 621010, P. R. China

${ }^{d}$ Key Laboratory of Solid Waste Treatment and Resource Recycle, Ministry of Education, State Key Laboratory Cultivation Base for Nonmetal Composites and Functional Materials, Southwest University of Science and Technology, Mianyang 621010, Sichuan, China

$\dagger$ Electronic supplementary information (ESI) available. See DOI: $10.1039 / \mathrm{c} 9 \mathrm{sc} 02202 \mathrm{c}$ oxide, molybdenum oxide $\left(\mathrm{MoO}_{3}\right)$, cadmium oxide, titanium dioxide, tungsten oxide, and zinc oxide. ${ }^{\mathbf{1 2 - 2 6}}$

As an important semiconductor material, $\mathrm{MoO}_{3}$ shows strong LSPR absorption after introduction of oxygen vacancies, aliovalent ions, and hydrogen ions, ${ }^{27}$ which induces a notable color change. Although there are some methods for preparation of plasmonic $\mathrm{MoO}_{3}$ nanomaterials, including $\mathrm{MoO}_{3-x}$, $\mathrm{H}_{1.68} \mathrm{MoO}_{3}, \mathrm{H}_{0.9} \mathrm{MoO}_{3}$ and $\mathrm{H}_{0.3} \mathrm{MoO}_{3},{ }^{16,28-30}$ most of them involve harsh reaction conditions (high temperature and pressure). Generally, the reported plasmonic $\mathrm{MoO}_{3}$ nanomaterials have blue color, and other colors are rarely obtained because of the lack of valid approaches to regulate their LSPRs. Moreover, the resulting plasmonic $\mathrm{MoO}_{3}$ nanomaterials are quite unstable, which are sensitive to oxidants and can be easily oxidized, ${ }^{18,28,31,32}$ leading to the disappearance of LSPR as the charge carrier density after oxidation is below the threshold to provide LSPR. Therefore, development of mild, facile, and reliable methods for preparation of stable plasmonic $\mathrm{MoO}_{3}$ nanomaterials with tunable color and LSPR properties is an urgent demand.

Here we report a facile and reliable strategy for the synthesis of oxygen-deficient $\mathrm{MoO}_{3}\left(\mathrm{MoO}_{3-x}\right)$ nanosheets using the dopamine serving as both the reductant and precursor for the formation of a polydopamine (PDA) surface coating (Scheme 1). The resulting $\mathrm{PDA}$-coated $\mathrm{MoO}_{3-x}$ nanosheets afford strong 

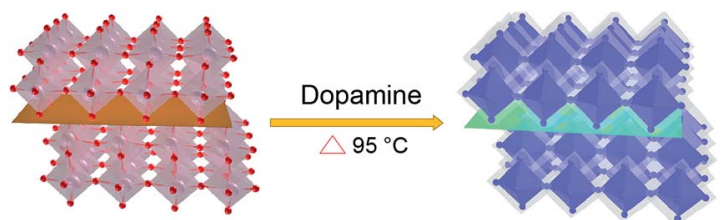

$\mathrm{MoO}_{3}$
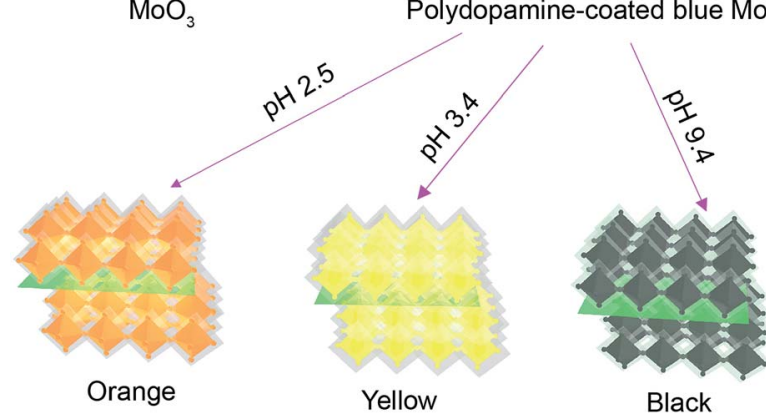

Scheme 1 Schematic illustration of the formation of multicolor PDAcoated $\mathrm{MoO}_{3-x}$ nanosheets with tunable plasmon resonance.

LSPR absorption, and their LSPR peaks are tuned from the ultraviolet to the near-infrared region (361-809 $\mathrm{nm}$ ) by altering the $\mathrm{pH}$ value of the medium, accompanying the production of variable colors (deep blue, faint bluish, orange, yellow and black). In addition, the stability of $\mathrm{MoO}_{3-x}$ nanosheets is studied in the presence of several oxidants. Furthermore, the plasmonic $\mathrm{MoO}_{3-x}$ nanosheets are employed as ultrasensitive surface-enhanced Raman scattering (SERS) substrates at femtomolar levels as well.

\section{Results and discussion}

\section{Structure analysis of PDA-coated $\mathrm{MoO}_{3-x}$ nanosheets}

The PDA-coated $\mathrm{MoO}_{3-x}$ nanosheets are synthesized by a redox reaction between $\mathrm{MoO}_{3}$ nanosheets and dopamine. Fig. 1a shows the transmission electron microscopy (TEM) image of $\mathrm{MoO}_{3-x}$ nanosheets, revealing a sheet morphology, and some large $\mathrm{MoO}_{3-x}$ nanosheets have a wrinkled surface, which has
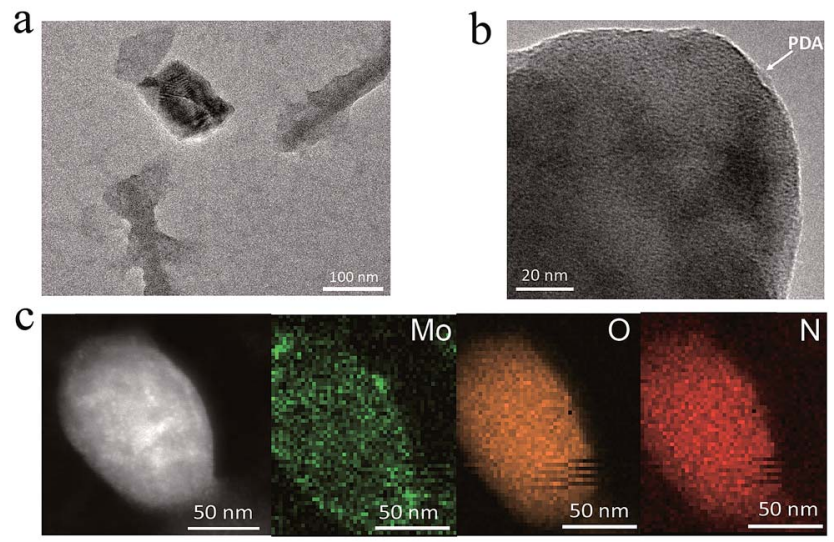

Fig. 1 Two-dimensional morphology of PDA-coated $\mathrm{MoO}_{3-x}$ nanosheets. (a) TEM, (b) HRTEM, and (c) STEM images and EDS elemental mapping profiles of PDA-coated $\mathrm{MoO}_{3-x}$ nanosheets. also been observed in other two-dimensional nanomaterials such as graphene oxide. ${ }^{33}$ From the high-resolution transmission electron microscopy (HRTEM) image of $\mathrm{MoO}_{3-x}$ nanosheets (Fig. 1b), it can be found that the nanosheets possess a double-layer structure, which corresponds to a dark $\mathrm{MoO}_{3-x}$ core and a PDA coating with a lower contrast surrounding the core. Conversely, there is no double-layer structure in pristine $\mathrm{MoO}_{3}$ nanosheets (Fig. S1†). To identify the elemental compositions of nanosheets and distribution of various elements, scanning transmission electron microscopy (STEM) and energy dispersive spectroscopy (EDS) mapping are conducted as shown in Fig. 1c. The resulting STEM image gives information regarding the generation of PDA-coated $\mathrm{MoO}_{3-x}$ nanosheets with the presence of a PDA shell. The EDS mapping confirms that $\mathrm{Mo}, \mathrm{O}$, and $\mathrm{N}$ elements are uniformly distributed throughout the nanosheets, in which the $\mathrm{N}$ element originates from the PDA molecule.

Characteristic infrared absorption bands of PDA-coated $\mathrm{MoO}_{3-x}$ nanosheets are shown in Fig. 2a, which are similar to that of pure PDA that is prepared by auto-oxidation of dopamine at $\mathrm{pH} 8.5$ and distinctly different from that of dopamine (Fig. S2 and $\mathrm{S} 3 \dagger$ ), confirming the presence of PDA on the surface of $\mathrm{MoO}_{3-x}$ nanosheets. The three absorption bands located at $3375 \mathrm{~cm}^{-1}, 2916 \mathrm{~cm}^{-1}$, and $1734 \mathrm{~cm}^{-1}$ are attributed to the $\mathrm{N}-$ $\mathrm{H}, \mathrm{C}-\mathrm{H}$, and $\mathrm{C}=\mathrm{O}$ stretching vibrations of PDA. The peaks appearing at $1628 \mathrm{~cm}^{-1}, 1478 \mathrm{~cm}^{-1}$, and $1247 \mathrm{~cm}^{-1}$ are ascribed to the benzene skeleton vibration of PDA. Also, the a
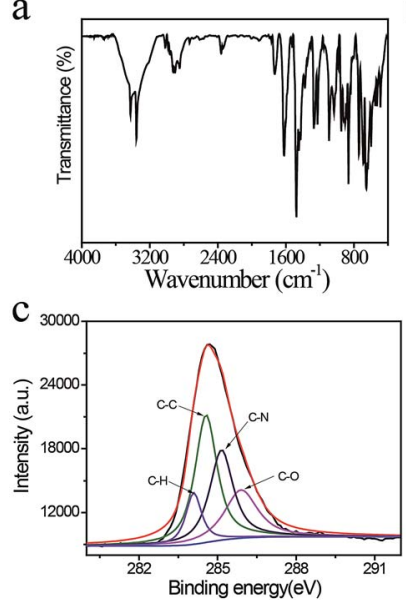

e

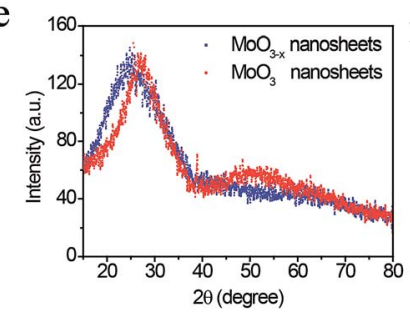

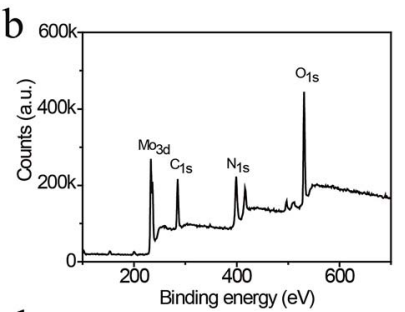
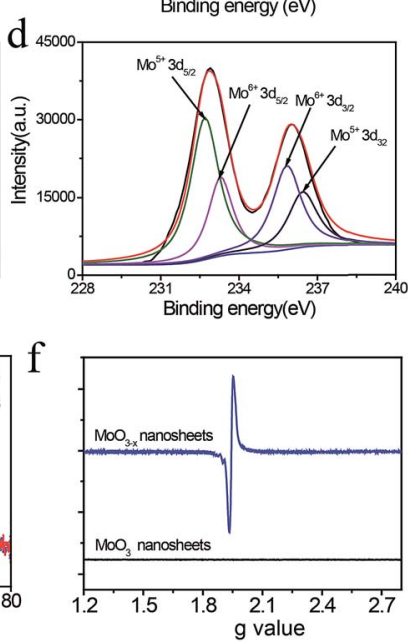

Fig. 2 Characterization of PDA-coated $\mathrm{MoO}_{3-x}$ nanosheets. (a) FT-IR spectrum and (b) survey XPS spectra of PDA-coated $\mathrm{MoO}_{3-x}$ nanosheets. (c) High-resolution XPS of $C$ 1s of PDA and (d) Mo 3d of PDAcoated $\mathrm{MoO}_{3-x}$ nanosheets. (e) XRD patterns and (f) EPR spectra of $\mathrm{MoO}_{3}$ and PDA-coated $\mathrm{MoO}_{3-x}$ nanosheets. 
characteristic peaks of $\mathrm{MoO}_{3-x}$ at $956 \mathrm{~cm}^{-1}$ (Mo-O stretching) and $870 \mathrm{~cm}^{-1}$ (Mo-O-Mo bridge stretching) are observed (Fig. 2a and S4 $\dagger$ ). The amount of PDA in the nanosheets is measured to be $7.3 \%$ by thermogravimetric analysis (TGA) as illustrated in Fig. S5. $\dagger$ The surface composition of PDA-coated $\mathrm{MoO}_{3-x}$ nanosheets is investigated using X-ray photoelectron spectra (XPS). The XPS signals of Mo, C, $\mathrm{N}$ and $\mathrm{O}$ elements are obtained (Fig. 2b), and the high-resolution spectra of the $\mathrm{C} 1 \mathrm{~s}$ in Fig. 2c can be fitted with four peaks, which are assigned to $\mathrm{C}-\mathrm{H}$, $\mathrm{C}-\mathrm{N}, \mathrm{C}-\mathrm{C}$, and $\mathrm{C}-\mathrm{O}$ species that demonstrate the presence of PDA (Fig. 2c).

In addition, the oxidation state of Mo is characterized by XPS as well. In contrast to the XPS spectra of $\mathrm{MoO}_{3}$ (Fig. S6 $†$ ), the PDA-coated $\mathrm{MoO}_{3-x}$ nanosheets include a certain amount of $\mathrm{Mo}^{5+}$ (Fig. 2d), suggesting that the $\mathrm{Mo}^{6+}$ is partially reduced to yield $\mathrm{Mo}^{5+}$, accompanying the removal of oxygen atoms with the assistance of hydrogen ions for the generation of water and oxygen vacancies. The crystal structure of blue PDA-coated $\mathrm{MoO}_{3-x}$ nanosheets is further studied. From the X-ray powder diffraction (XRD) patterns in Fig. 2e it can be seen that both $\mathrm{MoO}_{3-x}$ and $\mathrm{MoO}_{3}$ nanosheets display a broad peak in the range of $15-35^{\circ}$, which can be indexed to the (040) plane of a$\mathrm{MoO}_{3}$ (JCPDS no. 05-0508). Compared with $\mathrm{MoO}_{3}$ nanosheets, the peak position of blue PDA-coated $\mathrm{MoO}_{3-x}$ nanosheets moves to a lower angle, implying the expansion of the van der Waals gap in the oxygen-deficient $\mathrm{MoO}_{3-x}$ nanosheets. The existence of oxygen vacancies leaves unpaired electrons on the surface of $\mathrm{MoO}_{3-x}$ nanosheets, which are capable of being resolved by electron paramagnetic resonance (EPR) spectra. As indicated in Fig. 2f, no direct EPR signal is found in the $\mathrm{MoO}_{3}$ nanosheets, while the PDA-coated $\mathrm{MoO}_{3-x}$ nanosheets exhibit a strong EPR signal $(g=1.94)$, which provides direct evidence for the existence of unsaturated Mo species and oxygen vacancies.

\section{Tunable LSPR of $\mathrm{MoO}_{3-x}$ nanosheets from the ultraviolet to the near-infrared region}

The optical properties of the PDA-coated $\mathrm{MoO}_{3-x}$ nanosheets are examined by UV-Vis-NIR spectroscopy. Fig. 3a shows the UVVis-NIR spectra and the corresponding photographs of $\mathrm{MoO}_{3}$ nanosheet and PDA-coated $\mathrm{MoO}_{3-x}$ nanosheet dispersions. The $\mathrm{MoO}_{3}$ nanosheet dispersion is colorless, and it does not exhibit any absorption band in the wavelength region from $400 \mathrm{~nm}$ to $900 \mathrm{~nm}$. However, the blue PDA-coated $\mathrm{MoO}_{3-x}$ nanosheet dispersion has an absorption peak at $809 \mathrm{~nm}$. The red shift of the observed peak takes place when the solvent refractive index is increased, and a linear relationship between absorption peaks and solvent refractive index is achieved (Fig. $3 \mathrm{~b}$ and $\mathrm{S} 7 \dagger$ ), testifying that this absorption peak is attributed to the LSPR. ${ }^{34}$

The LSPR absorption band of PDA-coated $\mathrm{MoO}_{3-x}$ nanosheets can be readily tuned by altering the $\mathrm{pH}$ values of the reaction solution. The reduction capacity of dopamine increases with the increase of the $\mathrm{pH}$ value (Fig. $\mathrm{S} 8 \dagger$ ), which can induce the formation of more oxygen vacancies and therefore results in higher free-electron densities. As depicted in Fig. $3 \mathrm{c}$ and d, the LSPR peak of PDA-coated $\mathrm{MoO}_{3-x}$ nanosheets undergoes
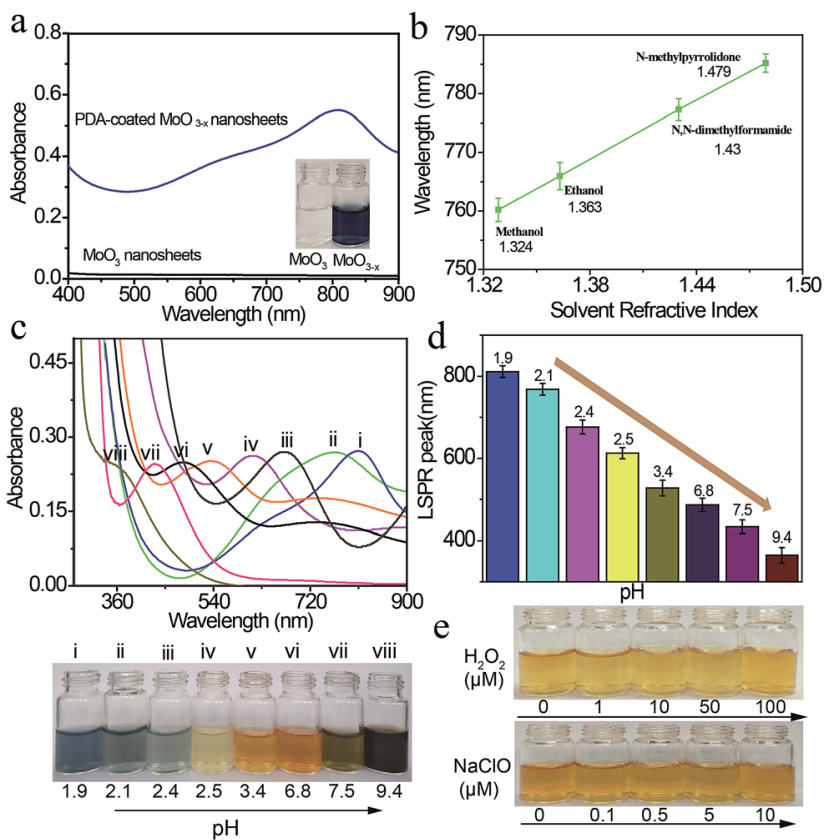

Fig. 3 Tunability of the plasmon resonance of PDA-coated $\mathrm{MoO}_{3-x}$ nanosheets. (a) UV-Vis-NIR spectra of $\mathrm{MoO}_{3}$ and PDA-coated $\mathrm{MoO}_{3-x}$ nanosheet dispersions. (b) LSPR peaks of the $\mathrm{MoO}_{3-x}$ nanosheet dispersion as a function of the solvent refractive index. (c and $d$ ) Dependence of UV-Vis-NIR spectra, and the corresponding photographs and LSPR peaks on the $\mathrm{pH}$ value of the reaction solution. (e) Photographs of a yellow $\mathrm{MoO}_{3-x}$ nanosheet dispersion in the absence and presence of $\mathrm{H}_{2} \mathrm{O}_{2}$ and $\mathrm{NaClO}$ with different concentrations.

a gradual blue shift from the NIR to the UV region $(809 \mathrm{~nm}$ to $361 \mathrm{~nm}$ ) as the $\mathrm{pH}$ value increases from 1.9 to 9.4. Correspondingly, the percentage concentration of $\mathrm{Mo}^{5+}$ determined by XPS increases from $42.86 \%$ to $59.94 \%$ (Fig. S9 $\dagger$ ), and there is no phase change when the LSPR peak of PDA-coated $\mathrm{MoO}_{3-x}$ shifts from $809 \mathrm{~nm}$ to $361 \mathrm{~nm}$ that is supported by TEM, XRD and ESR (Fig. S10-S12†). Interestingly, the blue shift of the LSPR peak creates a series of multicolor dispersions, including deep blue, faint bluish, orange, yellow and black. The LSPR peak shift spans as much as $448 \mathrm{~nm}$, which has not been realized in reported plasmonic doped metal oxide nanomaterials and challenges traditional noble metal nanocrystals as well. The blue shift is due to the higher free-electron densities based on the Drude model, ${ }^{16}$ and the highest charge carrier density is determined to be $1.32 \times 10^{21} \mathrm{~cm}^{-3}$ in the yellow $\mathrm{MoO}_{3-x}$ nanosheets (Fig. S13†). The chemical stability of the plasmonic PDA-coated $\mathrm{MoO}_{3-x}$ nanosheets is also evaluated. Because the oxygen vacancies have great affinity for oxygen molecules, the $\mathrm{MoO}_{3-x}$ nanosheets without a surface coating are trivially easy to be oxidized, causing the disappearance of LSPR thanks to the decrease of free-electron densities. The $\mathrm{MoO}_{3-x}$ nanosheets prepared using other reducing agents such as sodium borohydride, ascorbic acid and glutathione become colorless in the presence of oxidants $\left(\mathrm{H}_{2} \mathrm{O}_{2}\right.$ and $\left.\mathrm{NaClO}\right)$, revealing that they are not stable (Fig. S14 and S15 $\dagger$ ). In contrast, the color of the PDAcoated $\mathrm{MoO}_{3-x}$ nanosheet dispersion still remains even if a high concentration of $\mathrm{H}_{2} \mathrm{O}_{2}$ and $\mathrm{NaClO}$ is introduced as shown in 
Fig. 3e, affirming the strong resistance of PDA-coated $\mathrm{MoO}_{3-x}$ nanosheets to oxidation. This good stability is ascribable to the PDA surface coating that prevents the contact between $\mathrm{MoO}_{3-x}$ and oxidation reagents and consumes oxidation species because of the existence of reducing hydroxyl groups in the molecular structure of PDA.

\section{SERS properties of $\mathrm{MoO}_{3-x}$ nanosheets and the enhancement mechanism}

Next, in order to explore the sensing application of PDA-coated $\mathrm{MoO}_{3-x}$ nanosheets, the SERS performance of $\mathrm{MoO}_{3-x}$ nanosheets is estimated by using R6G as a model analyte. The R6G solution $\left(10^{-7} \mathrm{M}\right)$ is mixed with blue PDA-coated $\mathrm{MoO}_{3-x}$ nanosheets and dried in air. Fig. 4a shows the Raman spectra of R6G on glass and blue $\mathrm{MoO}_{3-x}$ nanosheet substrates. Under $514.5 \mathrm{~nm}$ laser light excitation, the four prominent characteristic scattering peaks of R6G at $612 \mathrm{~cm}^{-1}\left(\mathrm{R}_{1}\right), 773 \mathrm{~cm}^{-1}\left(\mathrm{R}_{2}\right)$, $1363 \mathrm{~cm}^{-1}\left(\mathrm{R}_{3}\right)$, and $1652 \mathrm{~cm}^{-1}\left(\mathrm{R}_{4}\right)$ can be discerned clearly on the blue $\mathrm{MoO}_{3-x}$ nanosheet substrate, whereas no detectable Raman signal is obtained on the glass substrate. These results illustrate that the blue PDA-coated $\mathrm{MoO}_{3-x}$ nanosheets are able to greatly enhance the Raman signal. To estimate the sensitivity of the SERS-active $\mathrm{MoO}_{3-x}$ nanosheets, a set of R6G solutions
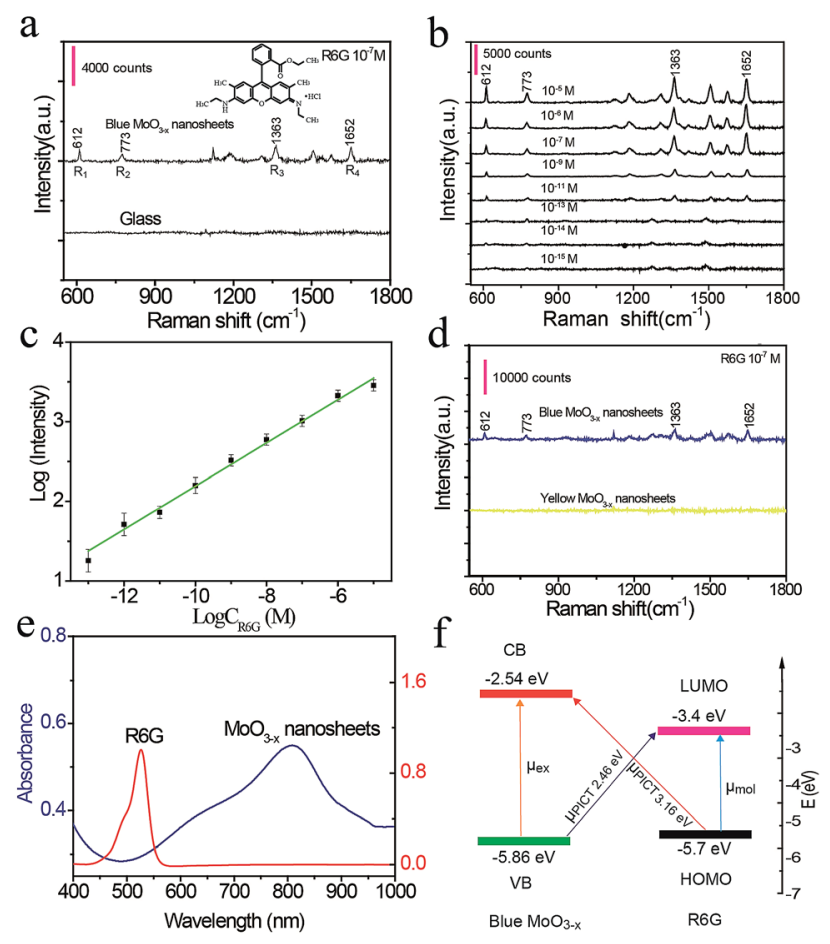

Fig. 4 SERS effects on the $\mathrm{MoO}_{3-x}$ nanosheets. (a) Raman spectra of R6G $\left(10^{-7} \mathrm{M}\right)$ on the glass and blue $\mathrm{MoO}_{3-x}$ nanosheet substrates. (b) SERS spectra of R6G adsorbed on the surface of blue $\mathrm{MoO}_{3-x}$ nanosheets with different concentrations in the range of $1 \mathrm{fM}$ to $10 \mu \mathrm{M}$. (c) The calibration curve for detection of R6G. (d) Comparison of the SERS effects of yellow and blue $\mathrm{MoO}_{3-x}$ nanosheets. (e) UV-Vis-NIR spectra of the blue $\mathrm{MoO}_{3}$ nanosheet dispersion and R6G solution. (f) Energylevel diagram and charge transfer transitions. $\mu_{\mathrm{ex}}$ and $\mu_{\mathrm{mol}}$ denote the electron excitation and molecular transition. $\mu_{\mathrm{PICT}}$ denotes the charge transfer transitions. with varying concentrations down to $1 \mathrm{fM}$ are prepared and tested as shown in Fig. $4 \mathrm{~b}$. The $\mathrm{R}_{2}$ fingerprint Raman band of R6G is still detectable at an ultralow concentration of $1 \mathrm{fM}$. The corresponding calibration curve for detection of R6G in the range of $10 \mu \mathrm{M}$ to $1 \mathrm{fM}$ is plotted as shown in Fig. 4c. The limit of detection is calculated to be $0.3 \mathrm{fM}$ based on $3 \sigma /$ slope ( $\sigma$ is the standard deviation of the blank, and the slope can be obtained from the calibration curve). This sensitivity is eight orders of magnitude superior to that of the $\mathrm{MoO}_{2}$ and $\mathrm{MoS}_{x} \mathrm{O}_{y}$-based SERS substrates for detection of $\mathrm{R} 6 \mathrm{G},{ }^{35,36}$ and it is comparable to or better than that of noble metal nanomaterial-based SERS substrates. ${ }^{37}$ What's more, the Raman enhancement factor (EF) reaches $1 \times 10^{10}$ at an R6G concentration of $10 \mathrm{pM}$. Meanwhile, several time-dependent consecutive peaks of R6G are also collected as shown in Fig. S16. $\dagger$ It can be seen that the characteristic peak of R6G $\left(R_{2}\right)$ at $1 \mathrm{fM}$ is clearly visible after irradiation with a laser for $1.5 \mathrm{~min}$, proving the acceptable reproducibility.

To understand the SERS mechanism, we compare the enhancement performance of yellow and blue PDA-coated $\mathrm{MoO}_{3-x}$ nanosheets (Fig. 4d). The results show that the yellow $\mathrm{MoO}_{3-x}$ nanosheets with high charge carrier density cannot boost the Raman signal. Likewise, the LSPR peak of blue PDAcoated $\mathrm{MoO}_{3-x}$ nanosheets does not overlap the absorption spectrum of R6G well as shown in Fig. 4e. On the basis of these results, the LSPR-induced electromagnetic enhancement mechanism is excluded. Alternatively, it has been reported that the chemical mechanism owing to the charge transfer between the SERS substrate and probe molecules is responsible for the semiconductor SERS effect. ${ }^{35,38,39}$ The two vibronically coupled lines at $612 \mathrm{~cm}^{-1}$ and $773 \mathrm{~cm}^{-1}$ are highly enhanced as depicted in Fig. 4a, suggesting that the charge transfer is a key factor for the SERS effect. More importantly, the energy levels of blue PDAcoated $\mathrm{MoO}_{3-x}$ nanosheets are determined by an electrochemical method using ferrocene as an internal standard (Fig. S17 and S18 $\dagger$ ). The calculated valence band (VB) and conduction band (CB) are $-5.86 \mathrm{eV}$ and $-2.54 \mathrm{eV}$, respectively (Fig. 4f). Simultaneously, the lowest unoccupied molecular orbital (LUMO) and highest occupied molecular orbital (HOMO) levels of R6G are $-3.4 \mathrm{eV}$ and $-5.7 \mathrm{eV} .{ }^{35}$ Consequently, there are two types of possible photo-induced charge transfer (PICT) processes from the VB to the LUMO at $2.46 \mathrm{eV}$ and from the HOMO to the $\mathrm{CB}$ at $3.16 \mathrm{eV}$ (Fig. 4f). The charge-transfer transition between the VB and the LUMO is near the laser light wavelength $(514.5 \mathrm{~nm}, 2.41 \mathrm{eV})$, which triggers charge transfer resonances. Apart from the charge transfer resonances, the molecular resonance at $514.5 \mathrm{~nm}$ laser light excitation overlaps the absorption cross section of R6G as well. In general, the charge transfer resonance is supposed to be greatly enhanced as it coupled with molecular resonance. ${ }^{38}$ Accordingly, the strong SERS effect of PDA-coated $\mathrm{MoO}_{3-x}$ nanosheets may originate from the coupling of charge transfer and molecular resonances.

\section{Conclusions}

In summary, we have developed a simple and reliable synthetic approach for preparation of plasmonic PDA-coated $\mathrm{MoO}_{3-x}$ 
nanosheets. The LSPR peak of the PDA-coated $\mathrm{MoO}_{3-x}$ nanosheets is controlled from $809 \mathrm{~nm}$ to $361 \mathrm{~nm}$ by changing the $\mathrm{pH}$ values of the reaction system. It is found that the formation of a PDA coating on the surface of $\mathrm{MoO}_{3-x}$ nanosheets is critical for the stability of the plasmonic properties. Remarkably, the present $\mathrm{MoO}_{3-x}$ nanosheets serve as a high-performance SERS substrate, which can be used for detection of Raman dyes at an $\mathrm{fM}$ level, and the $\mathrm{EF}$ value is as high as $1 \times 10^{10}$. The outstanding SERS effect of PDA-coated $\mathrm{MoO}_{3-x}$ nanosheets may arise from the coupling of charge transfer and molecular resonances. The current work is the first example of well-defined LSPR-tunable $\mathrm{MoO}_{3-x}$ nanosheets from the NIR to the UV region. The strategy and results offer a new avenue for preparation of plasmonic $\mathrm{MoO}_{3-x}$ nanosheets with good stability which have vast application prospects, including ultrasensitive detection, catalysis, light harvesting, and imaging.

\section{Experimental section}

\section{Materials}

$\mathrm{MoO}_{3-x}$ powers were purchased from Chron Chemicals. Dopamine, rhodamine $6 \mathrm{G}$, ascorbic acid, ferrocene, sodium sulfide, glutathione, $\mathrm{N}, \mathrm{N}$-dimethylformamide, $\mathrm{N}$-methylpyrrolidone, sodium hydroxide, and hydrochloric acid were obtained from Aladdin Chemical Reagent Co. Ltd. Ethanol, methanol, isopropanol, ethylene glycol, and other chemicals were purchased from Sinopharm Chemical Reagent Co. Ltd.

\section{Synthesis of $\mathrm{MoO}_{3}$ nanosheets}

The $\mathrm{MoO}_{3-x}$ nanosheets are synthesized by a reported method

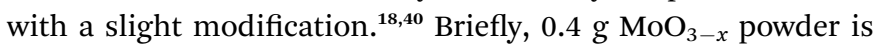
ground for $1 \mathrm{~h}$. Then, the resulting powder is dispersed in an ethanol/deionized (DI) water mixture $(26.4 \mathrm{~mL}$ ethanol and $23.6 \mathrm{~mL}$ deionized water) by ultra-sonication for $2 \mathrm{~h}(100 \mathrm{~W})$, followed by centrifugation at $8000 \mathrm{rpm}$ for $30 \mathrm{~min}$ at atmospheric temperature. The supernatant containing twodimensional $\mathrm{MoO}_{3-x}$ nanosheets $\left(3 \mathrm{mg} \mathrm{mL}^{-1}\right)$ is collected.

\section{Synthesis of multicolor plasmonic $\mathrm{MoO}_{3-x}$ nanosheets}

The blue $\mathrm{MoO}_{3-x}$ nanosheets are firstly synthesized. In a typical synthesis, $0.8 \mathrm{~mL}$ dopamine solution $\left(4 \mathrm{mg} \mathrm{mL}^{-1}\right)$ is added to $4 \mathrm{~mL} \mathrm{MoO}_{3-x}$ nanosheet dispersion $\left(3 \mathrm{mg} \mathrm{mL}^{-1}\right)$. Next, $0.6 \mathrm{~mL}$ $3 \mathrm{M} \mathrm{HCl}$ solution is injected into the above mixture and heated for $8 \mathrm{~h}$ in a water bath at $95^{\circ} \mathrm{C}$. For the synthesis of plasmonic $\mathrm{MoO}_{3-x}$ nanosheets with other colors, different volumes of $\mathrm{NaOH}$ solution $(1 \mathrm{mM})$ are used to adjust the $\mathrm{pH}$ values of the blue $\mathrm{MoO}_{3-x}$ nanosheet dispersion. The $\mathrm{MoO}_{3-x}$ nanosheet dispersions with different LSPR peaks at $809 \mathrm{~nm}, 768 \mathrm{~nm}$, $674 \mathrm{~nm}, 613 \mathrm{~nm}, 534 \mathrm{~nm}, 485 \mathrm{~nm}, 432 \mathrm{~nm}$, and $361 \mathrm{~nm}$ can be obtained using different $\mathrm{pH}$ values, during which different colors are produced.

\section{Characterization}

TEM and HRTEM were carried out on a FEI TECNAI G2 F20 microscope. XRD patterns were obtained on a D/MAX-IIIA X-ray diffractometer. UV-Vis-NIR absorption spectra were collected using a Shimadzu UV-1800 spectrometer. XPS was performed with an ESCALAB 250 photoelectron spectrometer. EPR spectra were obtained using a Bruker I200 spectrometer. The electrochemical measurements were performed using a CHI660C workstation. Fourier transform infrared spectra (FTIR) were obtained on a TENSOR spectrometer. TGA was performed on a TA Discovery thermal analysis system.

\section{Surface-enhanced Raman scattering tests}

To examine the Raman enhancement effect of $\mathrm{MoO}_{3-x}$ nanosheets, R6G is dissolved in DI water as the model molecule to prepare a series of standard R6G solutions with concentrations ranging from $1 \mathrm{fM}$ to $10 \mu \mathrm{M}$. The $\mathrm{MoO}_{3-x}$ nanosheet dispersion $\left(0.57 \mathrm{mg} \mathrm{mL}^{-1}\right)$ is mixed with the standard R6G solutions for $30 \mathrm{~min}$ and dried at room temperature. Subsequently, Raman spectra are collected, and the excitation wavelength of the laser is $514.5 \mathrm{~nm}$. The average density of R6G on the surface is calculated to be $3 \times 10^{16}$ molecules per $\mathrm{cm}^{2}$.

\section{Conflicts of interest}

The authors declare no conflict of interest.

\section{Acknowledgements}

We gratefully acknowledge the financial support from the National Natural Science Foundation of China (Grant No. 2170513, 441872039, and 41831285) and Longshan Academic Talent Research Supporting Program of SWUST (Grant No. 18LZX204 and 17LZX449).

\section{Notes and references}

1 J. R. Mejia-Salazar and O. N. Oliveira Jr, Chem. Rev., 2018, 118, 10617-10625.

2 P. Christopher, H. Xin and S. Linic, Nat. Chem., 2011, 3, 467472.

3 Y. Fang, W. Wang, X. Wo, Y. Luo, S. Yin, Y. Wang, X. Shan and N. Tao, J. Am. Chem. Soc., 2014, 136, 12584-12587.

4 R. F. Oulton, V. J. Sorger, D. A. Genov, D. F. P. Pile and X. Zhang, Nat. Photonics, 2008, 2, 496-500.

5 Y. He, B. Xu, W. Li and H. Yu, J. Agric. Food Chem., 2015, 63, 2930-2934.

6 J. Yang, Y. Guo, W. Lu, R. Jiang and J. Wang, Adv. Mater., 2018, 30, 1802227.

7 J. Olson, A. Manjavacas, L. Liu, W. S. Chang, B. Foerster, N. S. King, M. W. Knight, P. Nordlander, N. J. Halas and S. Link, Proc. Natl. Acad. Sci. U. S. A., 2014, 111, 14348-14353.

8 K. Kumar, H. Duan, R. S. Hegde, S. C. W. Koh, J. N. Wei and J. K. W. Yang, Nat. Nanotechnol., 2012, 7, 557-561.

9 A. Liu, G. Wang, F. Wang and Y. Zhang, Coord. Chem. Rev., 2017, 336, 28-42.

10 H. Chen, L. Shao, Q. Li and J. Wang, Chem. Soc. Rev., 2013, 42, 2679-2724.

11 L. Zhang, K. Xia, Z. Lu, G. Li, J. Chen, Y. Deng, S. Li, F. Zhou and N. He, Chem. Mater., 2014, 26, 1794-1798. 
12 X. Liu, H. Kang, H. Yuan, J. Park, S. J. Kim, Y. Cui, H. Y. Hwang and M. L. Brongersma, Nat. Nanotechnol, 2017, 12, 866.

13 P. Guo, R. D. Schaller, J. B. Ketterson and R. P. H. Chang, Nat. Photonics, 2016, 10, 267.

14 Y. Yang, Y. Yang, S. Chen, Q. Lu, L. Song, Y. Wei and X. Wang, Nat. Commun., 2017, 8, 1559.

15 M. Kanehara, H. Koike, T. Yoshinaga and T. Teranishi, J. Am. Chem. Soc., 2009, 131, 17736-17737.

16 H. Cheng, M. Wen, X. Ma, Y. Kuwahara, K. Mori, Y. Dai, B. Huang and H. Yamashita, J. Am. Chem. Soc., 2016, 138, 9316-9324.

17 W. Huang, J. Wang, J. Du, Y. Deng and Y. He, Microchim. Acta, 2019, 186, 79.

18 M. Li, X. Huang and H. Yu, Mater. Sci. Eng., C, 2019, 101, 614-618.

19 Y. Yue and Y. He, Anal. Sci., 2019, 35, 159-163.

20 J. Du, M. Zhao, W. Huang, Y. Deng and Y. He, Anal. Bioanal. Chem., 2018, 410, 4519-4526.

21 Y. Yang, K. Kelley, E. Sachet, S. Campione, T. S. Luk, J. P. Maria, M. B. Sinclair and I. Brener, Nat. Photonics, 2017, 11, 390.

22 E. Sachet, C. T. Shelton, J. S. Harris, B. E. Gaddy, D. L. Irving, S. Curtarolo, B. F. Donovan, P. E. Hopkins, P. A. Sharma, A. L. Sharma, J. Ihlefeld, S. Franzen and J. P. Maria, Nat. Mater., 2015, 14, 414-420.

23 T. R. Gordon, M. Cargnello, T. Paik, F. Mangolini, R. T. Weber, P. Fornasiero and C. B. Murray, J. Am. Chem. Soc., 2012, 134, 6751-6761.

24 K. Manthiram and A. P. Alivisatos, J. Am. Chem. Soc., 2012, 134, 3995-3998.

25 C. N. Valdez, A. M. Schimpf, D. R. Gamelin and J. M. Mayer, J. Am. Chem. Soc., 2016, 138, 1377-1385.

26 X. Zhang, M. He, P. He, C. Li, H. Liu, X. Zhang and Y. Ma, Appl. Surf. Sci., 2018, 433, 419-427.
27 M. M. Y. A. Alsaif, K. Latham, M. R. Field, D. D. Yao, N. V. Medhekar, G. A. Beane, R. B. Kaner, S. P. Russo, J. Z. Ou and K. Kalantar-zadeh, Adv. Mater., 2014, 26, 39313937.

28 H. Cheng, X. Qian, Y. Kuwahara, K. Mori and H. Yamashita, Adv. Mater., 2015, 27, 4616-4621.

29 H. S. Kim, J. B. Cook, H. Lin, J. S. Ko, S. H. Tolbert, V. Ozolins and B. Dunn, Nat. Mater., 2017, 16, 454.

30 W. Liu, Q. Xu, W. Cui, C. Zhu and Y. Qi, Angew. Chem., Int. Ed., 2017, 56, 1600-1604.

31 B. Y. Zhang, A. Zavabeti, A. F. Chrimes, F. Haque, L. A. O'Dell, H. Khan, N. Syed, R. Datta, Y. Wang, A. S. R. Chesman, T. Daeneke, K. Kalantar-zadeh and J. Z. Ou, Adv. Funct. Mater., 2018, 28, 1706006.

32 G. Song, J. Hao, C. Liang, T. Liu, M. Gao, L. Cheng, J. Hu and Z. Liu, Angew. Chem., Int. Ed., 2016, 55, 2122-2126.

33 O. C. Compton, S. Kim, C. Pierre, J. M. Torkelson and S. T. Nguyen, Adv. Mater., 2010, 22, 4759-4763.

34 S. H. Lee, H. Nishi and T. Tatsuma, Chem. Commun., 2017, 53, 12680-12683.

35 Z. Zheng, S. Cong, W. Gong, J. Xuan, G. Li, W. Lu, F. Geng and Z. Zhao, Nat. Commun., 2017, 8, 1993.

36 Q. Zhang, X. Li, W. Yi, W. Li, H. Bai, J. Liu and G. Xi, Anal. Chem., 2017, 89, 11765-11771.

37 D. K. Lim, K. S. Jeon, J. H. Hwang, H. Kim, S. Kwon, Y. D. Suh and J. M. Nam, Nat. Nanotechnol., 2011, 6, 452-460.

38 L. Tao, K. Chen, Z. Chen, C. Cong, C. Qiu, J. Chen, X. Wang, H. Chen, T. Yu, W. Xie, S. Deng and J. Xu, J. Am. Chem. Soc., 2018, 140, 8696-8704.

39 Q. Zhang, X. Li, Q. Ma, Q. Zhang, H. Bai, W. Yi, J. Liu, J. Han and G. Xi, Nat. Commun., 2017, 8, 14903.

40 M. M. Y. A. Alsaif, K. Latham, M. R. Field, D. D. Yao, N. V. Medehkar, G. A. Beane, R. B. Kaner, S. P. Russo, J. Z. Ou and K. Kalantar-zadeh, Adv. Mater., 2014, 26, 39313937. 